\title{
Oesophageal stricture associated with Crohn's disease
}

\author{
N. H. DYER, P. L. COOK, AND R. A. KEMP HARPER \\ From St Bartholomew's Hospital, London
}

Regional enteritis was originally described as a disease of the terminal ileum (Crohn, Ginzburg, and Oppenheimer, 1932). This concept of the condition has gradually been broadened and it is now realized that similar histopathological lesions may involve any part of the alimentary tract from stomach to anus (Crohn and Yunich, 1941; Lockhart-Mummery and Morson, 1960; Wolf and Marshak, 1962; Pryse-Davies, 1964). Many authors also mention that Crohn's disease may occasionally involve the oesophagus (Crohn and Yarnis, 1958), but there are very few case reports in the literature and other authorities doubt whether such lesions are really manifestations of Crohn's disease (Cooke, 1955).

We report two patients with Crohn's disease in whom radiological and endoscopic abnormalities of the lower oesophagus were found. In one of these patients surgical resection was performed thus providing full-thickness sections of the wall for histological examination.

\section{CASE REPORTS}

CASE 1 (J.W.) In 1946, when aged 36, this man developed recurrent ulceration of the mouth. Each attack commenced as crops of vesicles which developed into ulcers up to 1 in. in diameter and healed after three to four weeks. The posterior pharynx and genitalia were not involved. He never developed rashes, ocular symptoms, or arthralgia.

Shortly after this he developed a depressive anxiety state with hypochondriasis and multiple dyspeptic symptoms. This became progressively more severe, requiring many admissions to hospital. Numerous investigations, which included repeated barium meal examinations, showed no abnormality.

In 1958 the aphthous ulceration became more severe. Examination revealed extensive buccal scarring and a biopsy showed subacute non-specific inflammation. The ulcers healed after a short systemic course of cortisone and subsequent exacerbations have been much less severe.

He was readmitted in 1962 with a two-month history of lower oesophageal dysphagia superimposed on his many other longstanding dyspeptic symptoms. Apart from depression and aerophagy, examination showed clubbing of the finger nails and tenderness in the epigastrium.

The results of relevant investigations were as follows: haemoglobin $11.0 \mathrm{~g}$ per $100 \mathrm{ml}$, MCHC $33 \%$, white cell count 4,100 per $\mathrm{cmm}$, differential count normal, ESR (Westergren) $27 \mathrm{~mm}$ in one hour; serum proteins $6.7 \mathrm{~g}$ per $100 \mathrm{ml}$ (serum albumin $3.7 \mathrm{~g}$ per $100 \mathrm{ml}$ ). The Wassermann reaction was negative.

Radiology A chest radiograph was normal. A barium swallow and meal showed persistent irregularity and narrowing of the lower oesophagus (Figs. 1 and 2). There was no evidence of hiatus hernia or reflux.

Endoscopy This showed firm irregularity of the oesophageal wall posteriorly and to the left, $4 \mathrm{~cm}$ above the cardia, with an intact mucosa.

Biopsy There was a chronic inflammatory infiltrate beneath a normal squamous epithelium.

Thoracotomy was performed (in November 1962) because of the possibility of carcinoma. A small hard mass was found above the cardia and a lower oesophagectomy performed. Histological examination showed an unusual chronic inflammatory lesion involving the oesophagus and gastro-oesophageal junction but no evidence of malignancy.

In August 1964 he attended St Bartholomew's Hospital complaining of recurrent vomiting which was increasing in severity. Investigations showed an iron-deficiency anaemia in which the haemoglobin values varied from 8.6 to $10.6 \mathrm{~g}$ per $100 \mathrm{ml}$. The ESR was $55 \mathrm{~mm}$ in one hour. Occult blood was present in the faeces. Absorption tests of iron and vitamin A were abnormal. A barium meal revealed a marked delay in gastric emptying.

It was thought that bilateral vagotomy had occurred during the oesophagectomy and this was now interfering with pyloric action. This was confirmed at laparotomy (August 1964) and a pyloroplasty was performed. At this operation, four inflamed segments with the typical macroscopic appearance of Crohn's disease were noted in the lower jejunum and ileum. The largest lesion was at the ileo-caecal junction and measured 3 to $4 \mathrm{~cm}$. The regional lymph nodes were also enlarged. No resection or biopsy was performed.

The operation relieved the vomiting but his other symptoms were unchanged. The anaemia and malabsorption of iron persisted, but the ESR became normal and occult blood disappeared from the faeces. Faecal fat excretion was normal. Barium studies (January 1965) no longer showed gastric stasis, but typical segmental changes of Crohn's disease were present in the small intestine. He improved slightly on prednisolone but shortly afterwards was transferred to permanent psychiatric care.

Pathology The resected specimen of the oesophagus measured $9 \mathrm{~cm}$ of which the distal $1 \mathrm{~cm}$ was lined by 


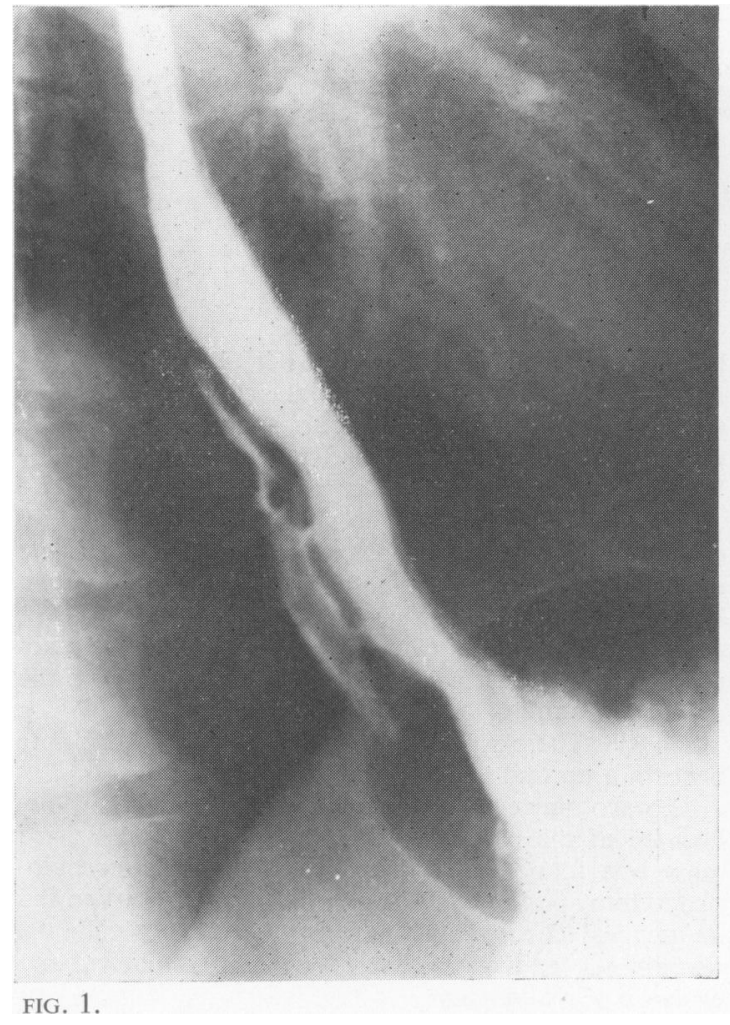

gastric mucosa. There was no mucosal ulceration. The muscle coat was markedly thickened to $0.6 \mathrm{~cm}$. Microscopy confirmed that the epithelium was intact. There was oedema and dense fibrosis in the submucosa which extended through the muscle to the outer connective tissue layer (Fig. 3). This was associated with numerous focal collections of plasma cells and lymphocytes (Figs. 4 and $5)$, which were often perivascular and sometimes perineural. The muscularis mucosa was normal. There was moderate lymphatic dilatation and slight hypertrophy of some myenteric nerve fibres. No giant cell lesions were present. The regional lymph nodes showed nonspecific chronic inflammation and prominent plasma cell production. These features indicate an unusual inflammatory stricture. There are no pathognomonic features of Crohn's disease but the general appearances are nevertheless consistent with this diagnosis. However, it should be noted that the lymphatic dilatation and lymphoid hyperplasia are less prominent than is usually seen in Crohn's disease elsewhere in the gut. There are no features to suggest other diagnoses such as arteritis, collagen disease, fungal infection, or stricture following intubation or gastrooesophagel reflux.

This man suffered from severe aphthous ulceration and then developed a lower oesophageal stricture of unusual histological appearance. Eighteen months later changes of Crohn's disease in the small in-

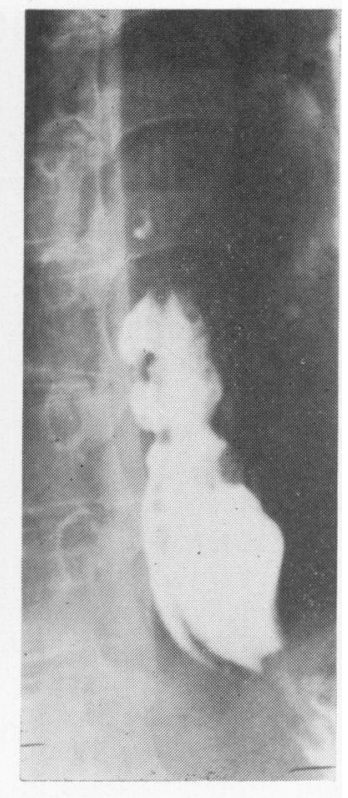

FIGS. 1 AND 2. Case 1: barium running in clefts in the lower oesophageal wall. Appearances suggest small breaks in the mucosa with deep infiltration (see discussion).

FIG. 2.

testine were discovered. Although there were no pathognomonic histological changes in the oesophagus, the picture was compatible with Crohn's disease, especially as no other causes of the lesion were apparent.

CASE 2 (L.B.) In 1947, when aged 24, this man developed recurrent ulceration of the mouth. Each crop of ulcers lasted about a week with recurrences every three weeks which he treated by simple oral hygiene until 1960 . The ulceration then suddenly became worse and he also developed a maculopapular rash on the trunk and legs. The ulcers and rash disappeared after treatment with prednisolone. The rash has never returned but the ulceration recurred almost at once and spread to the throat. It again responded to prednisolone but relapsed quickly and the patient was admitted to St Bartholomew's Hospital.

He was unable to open his mouth, speak, or swallow solids. He had also experienced night sweats, lassitude, slight loss of weight, and an aching epigastric pain during the three weeks before admission. On examination he was pale and had an irregular fever to $101 \cdot 8^{\circ} \mathrm{F}\left(38 \cdot 8^{\circ} \mathrm{C}\right)$. Large confluent ulcers involved the whole of his mouth and several shallow painless ulcers were present on the scrotum. The liver was palpable $2 \mathrm{~cm}$ below the costal margin. A diagnosis of Behcet's syndrome was made, although he never suffered from ocular, joint, neuro- 


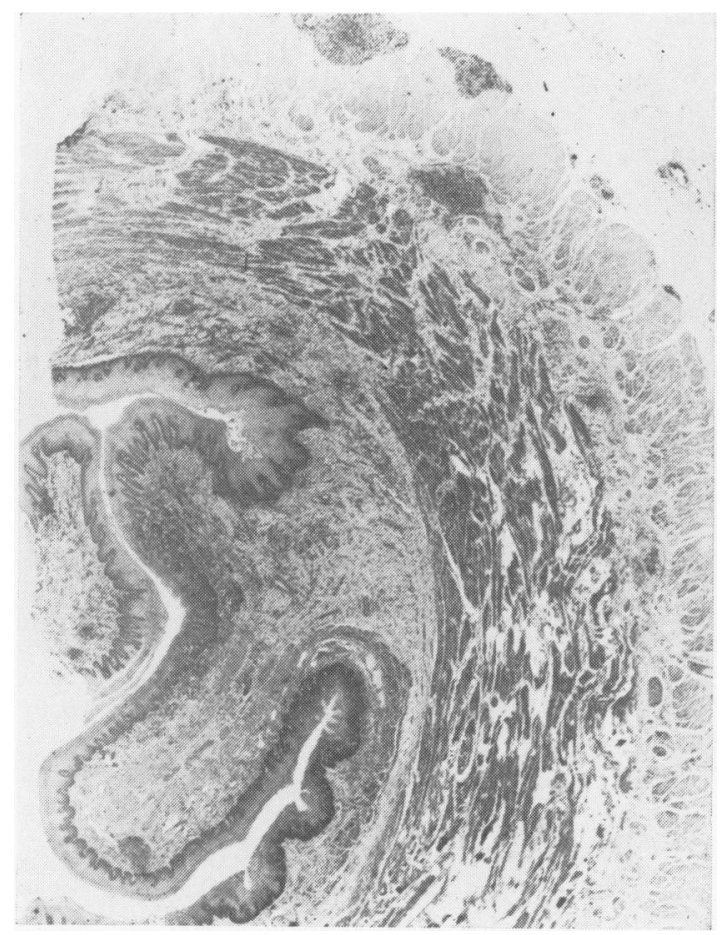

FIG. 3.

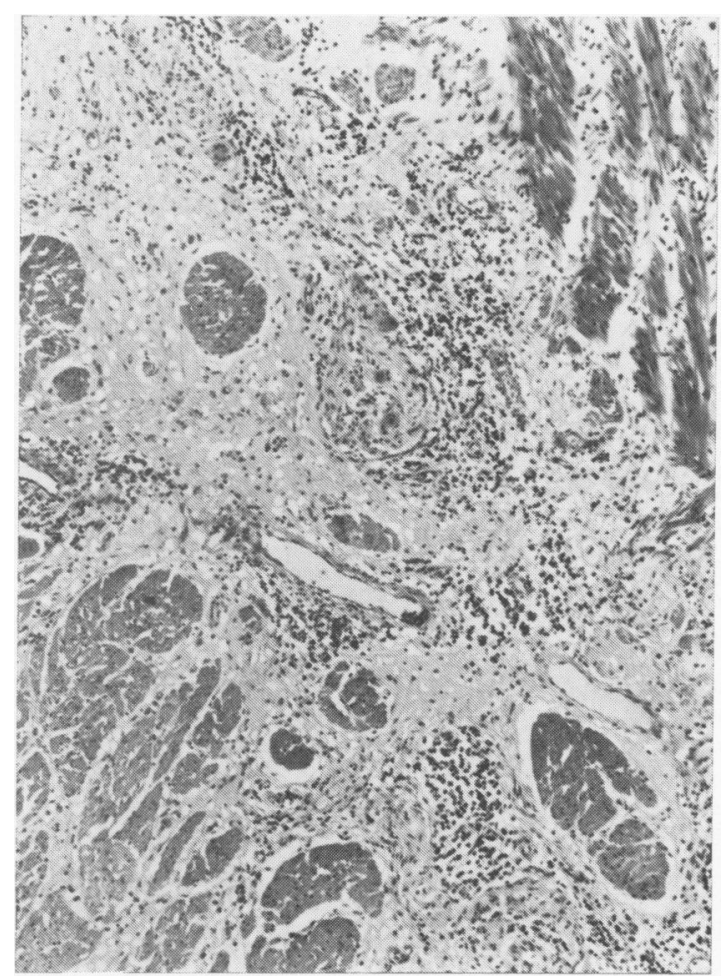

FIG. 4.

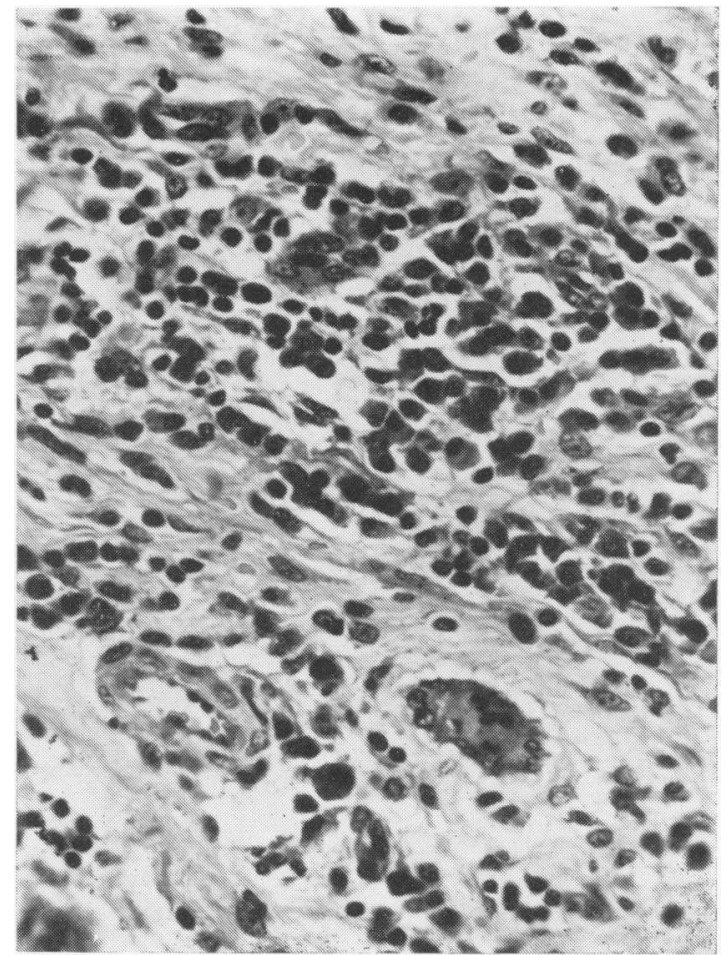

FIG. 5 .

FIG. 3. Case 1: oesophagectomy specimen. Haematoxylin and eosin $\times 10$.

FIGS. 4 and 5. Case 1: inflammatory infiltration of the oesophagus. Haematoxylin and eosin $\times 60$ (Fig. 4) and $\times 300$ (Fig. 5). 


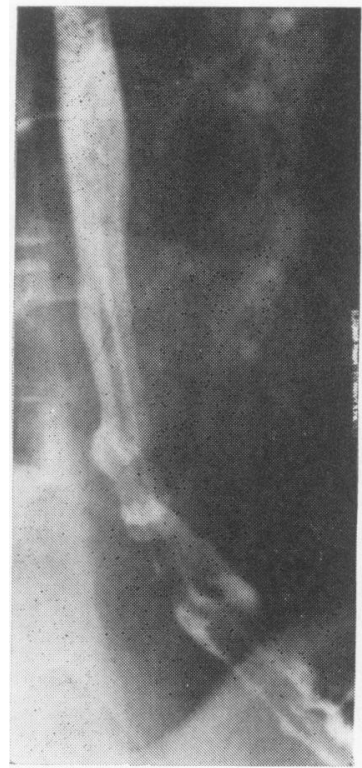

FIG. 6 .

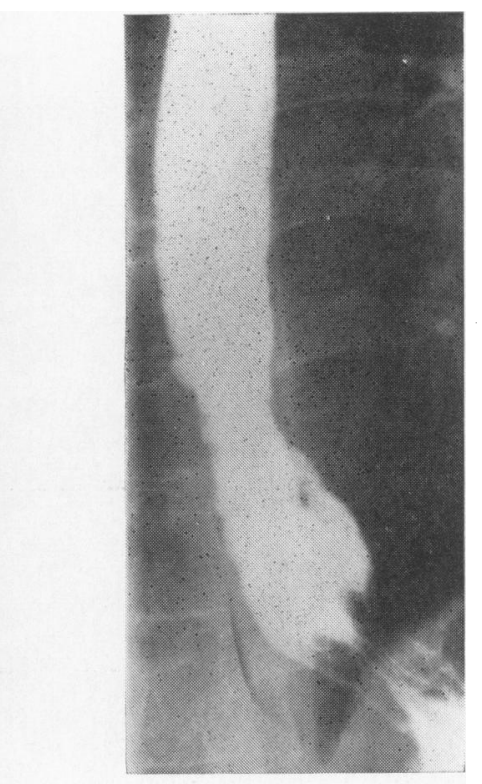

FIG. 7.
FIGs. 6 and 7. Case 2: barium swallow showing lower oesophagus. logical, cardiovascular, or pulmonary complications. Papules or pustules have never been observed at injection sites.

Investigations were as follows: haemoglobin $8.0 \mathrm{~g}$ per $100 \mathrm{ml}$, MCHC $29.5 \%$, white cell count 18,000 per cmm containing $82 \%$ neutrophils, ESR (Westergren) $118 \mathrm{~mm}$ in one hour; serum proteins $6.8 \mathrm{~g}$ per $100 \mathrm{ml}$ (serum albumin $4.4 \mathrm{~g}$ per $100 \mathrm{ml}$ ). Electrophoresis showed an increase in $\alpha_{2}$ globulin.

On the fourth day after admission he collapsed with severe abdominal pain, and a laparotomy (April 1961) showed Crohn's disease of the distal 18 inches of the ileum with a perforation 12 inches from the ileocaecal valve. The perforation was oversewn and an ileotransverse colostomy was constructed which bypassed the terminal $2 \mathrm{ft}$ of ileum. He made a rapid recovery and the oral ulcers again healed while receiving steroids to cover the operation. A nasogastric tube was in position for several days after the perforation had occurred.

One month later, while awaiting elective resection of the diseased segment of ileum, he passed several melaena stools. The bypassed bowel was therefore resected immediately (June 1961) and the diagnosis of Crohn's disease was confirmed histologically.

The mouth ulcers recurred postoperatively and systemic treatment with prednisolone in minimal maintenance dosage has been necessary ever since. From this time, besides suffering from persistent slight diarrhoea containing mucus, he has also developed occasional upper abdominal discomfort during meals and on four or five occasions has noticed a very mild sensation of food sticking behind the lower sternum. Barium studies were normal in 1962, but in 1965, although there was no evidence of ileitis, irregularity of the lower oesophagus had developed with the barium running in deep clefts in the wall. These changes showed some progression in 1967 and he was admitted for further investigation in May 1968 at which time he had developed a slightly more prolonged bout of dysphagia lasting several days. His general health was good. Physical examination was normal except for severe buccal scarring and two small aphthous ulcers. The scrotum was slightly scarred.

Investigations showed that his haematological and nutritional status was normal. Tests of small intestinal function were not impaired.

Radiology A barium swallow again showed a bizarre lesion at the lower end of the oesophagus (Figs. 6 and 7). There was no evidence of hiatus hernia or reflux. Cineradiography (Dr D. A. W. Edwards) confirmed the presence of a lower oesophageal stricture which did not behave like a typical intubation stricture.

Endoscopy The mucosal folds of the lower oesophagus were thickened and oedematous with some bluish discoloration. The segment was not rigid and showed no oesophagitis or ulceration.

Pathology The resected specimen of the small intestine consisted of $42 \mathrm{~cm}$ of terminal ileum and $18 \mathrm{~cm}$ of caecum and ascending colon. The distal $30 \mathrm{~cm}$ of ileum showed thickening of the wall with areas of hyperplasia and atrophy of the mucosa. Microscopy revealed areas of active ulceration in an otherwise intact mucosa together with submucosal oedema, fibrosis, and infiltration with chronic inflammatory cells. The fibrosis and inflammation extended into the muscle coat. No giantcell granulomata were seen. The appearances were fully consistent with Crohn's disease.

Three biopsies of the oesophagus were obtained with a Crosby capsule. The mucosa was intact, but the sub- 
mucosa showed oedema and non-specific chronic inflammatory changes. The inflammatory infiltration varied from slight to considerable and consisted of lymphocytes and plasma cells with increased numbers of eosinophils in two specimens.

This man had recurrent aphthous ulceration for years. After a severe episode of mucocutaneous ulceration he developed a perforation of the ileum and Crohn's disease was discovered at laparotomy. He subsequently experienced dysphagia. This could have been related to a short period of nasogastric intubation, but there was no radiological abnormality present one year after intubation and the later appearances are unlike those of an intubation stricture. Pathological examination of the entire thickness of the oesophageal wall has not been performed.

\section{DISCUSSION}

Heffernon and Kepkay (1954) described a man who developed a lower oesophageal polyp in association with increasing coarseness of the mucosal folds of the stomach, duodenum, and jejunum on radiography. Biopsy of the polyp showed only non-specific submucosal inflammation, and the polyp disappeared with intensive antacid therapy, although the radiological changes in the stomach and small intestine worsened. Two large penetrating ulcers developed in the distal duodenum and were complicated by haemorrhage which necessitated resection of the upper small intestine. However, melaena recurred within a few days and the patient died. Although the upper jejunum was thickened, histology showed non-specific inflammatory changes only and this was mild except in relation to the ulcers. The authors attributed all the lesions to regional enteritis, but the clinical course was unlike that of Crohn's disease and the pathological changes were nonspecific at all sites.

Recently, Gelfand and Krone (1968) have described a man who complained of symptoms of Crohn's disease of the colon for three months and then developed dysphagia. Barium studies and oesophageal manometry were normal but endoscopy showed diffuse dusky hyperaemia and oedema of the lower two-thirds of the oesophagus and biopsy indicated mild focal submucosal oesophagitis. The dysphagia responded dramatically to prednisone, but an ulcer developed in the mid-oesophagus which eventually healed on high doses of steroids. No further histology was obtained, but the authors considered that the oesophageal lesion was due to Crohn's disease in view of the response to steroid therapy and the failure to demonstrate any other cause. Oro-genital ulceration was not mentioned in this report.
Such cases are extremely rare in the literature and the incidence of any form of oesophagael involvement must be very low. On reviewing 12 of the largest reported series of Crohn's disease in which the site of the lesions was analysed, we were unable to find any cases with oesophageal abnormalities out of a total of 2,520 cases. Marshak (1964) and Crohn (1968, personal communication) both state that they have never seen a case. However, two cases of ulcerative oesophagitis complicating non-specific ulcerative colitis have recently been described (Kundsen and Sparberg, 1967; Rosendorff and Grieve, 1967). Our two cases were drawn from a total of 198 patients seen over an 11-year period.

It is not possible to delineate a clinical picture on the basis of so few reported cases, especially when the histological diagnosis is not final. Investigation will usually depend on radiology and endoscopy, both of which have distinct limitations. However, if either of these techniques demonstrate an unusual lesion in the oesophagus in a patient with established Crohn's disease elsewhere, a skip lesion should be considered. In the absence of bowel lesions elsewhere, the diagnosis is unlikely to be suggested but it might be rewarding to consider Crohn's disease in the differential diagnosis of any atypical stricture. Endoscopy may show thickened folds or a more definite mass and will exclude ulcerative conditions and reflux oesophagitis. Biopsy can only be pathognomonic if giant cell lesions are found, but these are not invariable features of Crohn's disease elsewhere (Hadfield, 1939) and their absence does not militate against the diagnosis. Transmural inflammation cannot be shown by biopsy and so the diagnosis rests on the presence of oedema, fibrosis, and chronic inflammation beneath an intact epithelium: features which are not specific. Surgical or necropsy specimens will be available only occasionally and even then diagnosis may be difficult, as in case 1 .

The radiological features were most unusual. Both patients showed disturbance and irregularity of the lower oesophagus with marked thickening of the mucosal folds. This was of such a degree in case 2 as to appear like multiple polypi. The filling of the clefts between the coarse mucosal folds could be regarded as due to ulceration or extravasation of the barium into the wall but this impression was not confirmed by the endoscopical and pathological demonstration of an intact mucosa. The transition from normal to abnormal oesophagus is gradual. The narrowed segment can be seen to vary in calibre during short periods of fluoroscopy. Although the passage of peristaltic waves through the segment is interrupted and the barium is slow to clear from the clefts, the passage of the main bulk of each bolus 
into the stomach is only very slightly delayed. The relative pliability of the segment is also seen at endoscopy and serves to differentiate from the fixed rigidity of carcinomatous infiltration or the late fibrotic phase of reflux oesophagitis or ingestion of corrosives. An abrupt shoulder deformity at the end of the stenosis has not been seen. The oesophageal wall can sometimes be outlined against the pleural reflection, the so-called 'oesophageal stripe', but this feature could not be seen and so increased thickness of the wall could not be demonstrated.

The radiological changes may be differentiated from those of oesophageal varices by their less serpiginous contour and lack of accentuation in the horizontal position and resting phase. Furthermore, a thin barium mixture, which facilitates the demonstration of varices, might more clearly differentiate between mucosal clefts and extravasation into the wall. Acute reflux oesophagitis, carcinoma, stricture after ingestion of corrosive substances, intramural rupture, tertiary contractions, and other disorders of motility all present characteristically different radiological patterns.

The association of oral ulceration in both of our patients is interesting and may reflect a predilection for involvement of the upper alimentary tract. It is just possible that they may have an altered tissue reactivity to insults in this area which result in lesions which are not specific and not primary Crohn's inflammation. Crohn's disease can be complicated by aphthous stomatitis which may sometimes be severe and is occasionally associated with genital ulcers (Forman, 1966). This raises the possibility that our patients had Behcet's syndrome which may be diagnosed in the absence of ocular lesions (Curth, 1946), and that the oesophageal lesions were manifestations of this syndrome. Painful dysphagia is common in Behcet's syndrome but is referred to the upper oesophagus. Involvement of the lower oesophagus has not been documented although gastrointestinal disturbances have been described (Oshima, Shimizu, Yokohori, Matsumoto, Kano, Kagami, and Nagaya, 1963). We have never seen radiological abnormalities in Behcet's syndrome which resemble those found in our two patients. Brombart (1961) does not consider Behcet's syndrome or Crohn's disease in the differential diagnosis of lower oesophageal lesions. The occurrence of ileal lesions in Behcet's syndrome (Bechgaard, 1940; Oshima et al, 1963) suggests that the association of mucocutaneous syndromes and Crohn's disease may be more frequent than is generally recognized.

\section{SUMMARY}

Unusual oesophageal strictures occurring in two cases of Crohn's disease are reported. The radiological findings were similar in both cases and are described together with some differentiating features. A survey of the literature indicates that comparable lesions occur very rarely. In one case oesophageal resection provided material for an adequate pathological examination, and the histological changes were consistent with Crohn's disease, although no absolute diagnostic features were present. The concept of Crohn's disease of the oesophagus and the difficulties in its diagnosis are discussed.

We are grateful to Dr D. A. W. Edwards and Dr A. M. Dawson for helpful criticism of this paper, and to the Board of Governors of St Bartholomew's Hospital and The Dowager Countess Eleanor Peel Research Fund for financial support.

\section{REFERENCES}

Bechgaard, P. (1940). Case of recurrent aphthous stomatitis accompanied by conjunctivitis and ulcerations of genitals and skin. (Danish). Ugeskr. Laeg., 102, 1019-1023.

Brombart, M. (1961). Clinical radiology of the oesophagus. J. Wright, Bristol.

Cooke, W. T.(1955). Nutritional and metabolic factors in the aetiology, and treatment of regional ileitis. Ann. roy. Coll. Surg. Engl., 17, $137-158$.

Crohn, B. B., Ginzburg, L., and Oppenheimer, G. D. (1932). Regional ileitis: a pathologic and clinical entity. J. Amer. med. Ass., 99, 1323-1329.

_- and Yarnis, H. (1958). Regional ileitis 2nd Ed., p.1. Grune and Stratton, New York.

and Yunich, A. M. (1941). Ileojejunitis. Ann. Surg., 113, 371-380.

Curth, H. O. (1946). Recurrent genito-oral aphthosis and uveitis with hypopyon. Arch. Derm. Syph. (Chic.), 54, 179-196.

Forman, L. (1966). The skin and the colon. Trans. St. John's Hosp. derm. Soc. (Lond.), 52, 139-154.

Gelfand, M. D., and Krone, C. L. (1968). Dysphagia and esophageal ulceration in Crohn's disease. Gastroenterology, 55, 510-514.

Hadfield, G. (1939). The primary histological lesion of regional ileitis. Lancet. 2, 773-775.

Heffernon, E. W., and Kepkay, P. H. (1954). Segmental esophagitis, gastritis and enteritis. Gastroenterology, 26, 83-88.

Kundsen, K. B., and Sparberg, M. (1967). Ulcerative esophagitis and ulcerative colitis. J. Amer. med. Ass., 201, 140.

Lockhart-Mummery, H. E., and Morson, B. C. (1960). Crohn's disease (regional enteritis) of the large intestine and its distinction from ulcerative colitis. Gut, 1, 87-105.

Marshak, R. H. (1964). In Gastro-enterology, edited by H. L. Bockus, 2nd ed. 2, p. 278. Saunders, Philadelphia.

Oshima, Y., Shimizu, T., Yokohari, R., Matsumoto, T., Kano, K., Kagami, T., and Nagaya, H. (1963). Clinical studies in Behcet's syndrome. Ann. rheum. Dis., 22, 36-45.

Pryse-Davies, J. (1964). Gastro-duodenal Crohn's disease. J. clin. Path., 17, 90-94.

Rosendorff, C., and Grieve, N. W. T. (1967). Ulcerative oesophagitis in association with ulcerative colitis. Gut, 8, 344-347.

Wolf, B. S., and Marshak, R. H. (1962). Granulomatous colitis (Crohn's disease of the colon). Roentgen features. Amer. J. Roentgenol., 88, 662-670. 